\title{
Helioseismology of Sunspots: Confronting Observations with Three-Dimensional MHD Simulations of Wave Propagation
}

\author{
R. Cameron • L. Gizon • T.L. Duvall Jr.
}

Received: 15 November 2007 / Accepted: 11 February 2008 / Published online: 22 March 2008

(C) The Author(s) 2008. This article is published with open access at Springerlink.com

\begin{abstract}
The propagation of solar waves through the sunspot of AR 9787 is observed by using temporal cross-correlations of SOHO/MDI Dopplergrams. We then use threedimensional MHD numerical simulations to compute the propagation of wave packets through self-similar magnetohydrostatic sunspot models. The simulations are set up in such a way as to allow a comparison with observed cross-covariances (except in the immediate vicinity of the sunspot). We find that the simulation and the $f$-mode observations are in good agreement when the model sunspot has a peak field strength of $3 \mathrm{kG}$ at the photosphere and less so for lower field strengths. Constraining the sunspot model with helioseismology is only possible because the direct effect of the magnetic field on the waves has been fully taken into account. Our work shows that the full-waveform modeling of sunspots is feasible.
\end{abstract}

Keywords Sun: helioseismology $\cdot$ Sun: sunspots $\cdot$ Sun: magnetic fields

\section{Introduction}

The subsurface magnetic structure of sunspots is poorly known. The theoretical picture is that sunspots are either monolithic or not (Parker, 1979), or change from being monolithic to "disconnected" over the course of the first few days of their lives (Schüssler and Rempel, 2005). The observational picture is limited because the subsurface structure is inherently difficult to infer. The most promising possibility by far is local helioseismology. Local helioseismology includes several techniques of data analysis, such as Fourier - Hankel analysis (e.g., Braun, 1995), time - distance analysis (e.g., Duvall et al., 1993), and helioseismic

Helioseismology, Asteroseismology, and MHD Connections Guest Editors: Laurent Gizon and Paul Cally.

R. Cameron $(\bowtie) \cdot$ L. Gizon

Max-Planck-Institut für Sonnensystemforschung, 37191 Katlenburg-Lindau, Germany

e-mail: cameron@mps.mpg.de

T.L. Duvall Jr.

Solar Physics Laboratory, NASA Goddard Space Flight Center, Greenbelt, MD 20771, USA 
holography (e.g., Lindsey and Braun, 1997). For example, the time - distance approach (Duvall et al., 1993) has been applied to determine wave-speed variations and flows associated with sunspots (e.g., Kosovichev, Duvall, and Scherrer, 2000; Zhao, Kosovichev, and Duvall, 2001; Couvidat, Birch, and Kosovichev, 2006). These inversions did not take into account the direct effects of the magnetic field. We do not mean to give the impression that such effects have not been investigated, but rather that they are only beginning to be incorporated into helioseismic inversions. Fourier-Hankel decompositions of the acoustic wave field ( $p$ modes) near sunspots by Braun, Duvall, and LaBronte (1987) showed that incoming $p$ modes are phase-shifted and "absorbed" by sunspots. This triggered many studies of the effects of the magnetic field on solar waves. For example, Spruit (1991) suggested that sunspot magnetic fields are responsible for the observed acoustic wave "absorption" by partially converting incoming $p$ modes into slow magnetoacoustic waves. This idea was followed up in detail by Spruit and Bogdan (1992), Cally and Bogdan (1993, 1997), Cally, Bogdan, and Zweibel (1994), Hindman, Zweibel, and Cally (1996), Bogdan and Cally (1997), and Rosenthal and Julien (2000). An important result was the realization that nonuniform and nonvertical magnetic fields are required to explain the observations. In particular, Cally (2000) reported on numerical calculations, showing that inclined magnetic fields are able to achieve levels of absorption compatible with the observations. This aspect of the problem was followed up by Cally, Crouch, and Braun (2003) and Crouch et al. (2005), who placed constraints on the strength of the sunspot's magnetic field. More recently, attention has been placed on upward propagating magnetoacoustic waves and their possible observational signatures (Schunker and Cally, 2006; Khomenko and Collados, 2006; Cally, 2007; Cally and Goossens, 2008). The use of direct numerical simulations in helioseismology, with or without magnetic fields, has also undergone rapid development. The aims of these simulations include validating helioseismic techniques (e.g., Zhao et al., 2007) and, more importantly, increasing our understanding of what the seismic observations reveal about the solar interior. Here we restrict our focus to simulations of linearized wave propagation through an inhomogeneous solar atmosphere. Examples of these linear wave propagation studies, most of which do not include the direct effects of magnetic fields, include Birch et al. (2001), Khomenko and Collados (2006), Hanasoge, Duvall, and Couvidat (2007), Hanasoge et al. (2006), and Hanasoge and Duvall (2007). Some results of these studies clearly have a large bearing on the correct interpretation of the helioseismic signatures of sunspots.

In this paper we first design a technique to image the interaction of waves with sunspots, using appropriate averaging of the cross-correlations of the random seismic wave field (SOHO/MDI data). We proceed further by using numerical simulations to do full-waveform forward modeling of the passage of solar waves through a sunspot. This is done with the SliM code (Cameron, Gizon, and Daiffallah, 2007), specifically developed for this purpose. The ultimate goal is to understand the observed cross-correlations in terms of the properties of our parametric sunspot models.

This paper is organized as follows. The SOHO/MDI observations of sunspot AR 9787 are presented in Section 2 and their helioseismic analysis in Section 3. We describe our numerical simulation code in Section 4. We compare the observations (cross-correlations) against the vertical velocity on a horizontal cut taken from the simulation at the height of the quiet-Sun photosphere. This, among other reasons, means the comparison is only meaningful outside the sunspot. Except in the immediate vicinity of the sunspot, the comparison between the observations and the simulations is very encouraging, as shown in Section 5. We place a helioseismic constraint on the sunspot magnetic field in Section 6. Our work strongly suggests that we will be able to use observations and simulations in combination to constrain the subsurface structure of sunspots by taking direct and indirect magnetic effects into account. 


\section{SOHO/MDI Observations of Active Region 9787}

The helioseismic analysis of a sunspot is more or less difficult depending on which sunspot is being studied. We have been searching the SOHO/MDI database for the ideal sunspot, that is, a sunspot that is isolated (does not belong to a complex sunspot group), has a simple geometry (circular shape), and evolves slowly in time as it moves across the solar disk. To find such a "theorist's sunspot," we searched the MDI Dynamics Program, which combines the advantage of good spatial resolution $\left(0.12^{\circ}\right.$ at disk center $)$ with a complete view of the solar disk. The full-disk Dopplergrams are available each minute during two to three months each year since 1996. The sunspot we have selected is that of Active Region 9787, continuously observed by MDI during nine days: 20-28 January 2002. The Dopplergrams were remapped by using Postel's azimuthal equidistant projection almost - but not exactly - centered on the sunspot, using a tracking angular velocity of $-0.1102^{\circ} \mathrm{day}^{-1}$ (in the Carrington frame). In addition to the Dopplergrams, we also used the line-of-sight magnetograms (each minute) and all the intensity images (one per six hours). The daily averages of these three quantities are shown in Figure 1. Apart from some plage, there is no other active region in the vicinity of the sunspot during the entire observation sequence. The sunspot of AR 9787 is large and quite stable over the nine days of the observations, even though it starts decaying from 27 January onward.

Using the MDI intensity images, we measured the center of the sunspot's umbra. As shown in Figure 2, the sunspot has a significant amount of proper motion. Thus we chose to split the time series into six-hour subsets and to analyze each subset separately. The images belonging to each particular subset were remapped into a new Postel map, with the center of the projection corresponding exactly to the center of the sunspot's umbra in the middle of the six-hour time interval. These 36 six-hour time series of Dopplergrams, centered on the sunspot's position, are the basic data that we used for the helioseismic analysis, which we discuss in the next section.

The average intensity image of the sunspot, corrected for the proper motion of the sunspot, is shown in Figure 3. From this average image, which is still sharp, we determine the average umbral and penumbral radii to be 9 and $20 \mathrm{Mm}$, respectively.

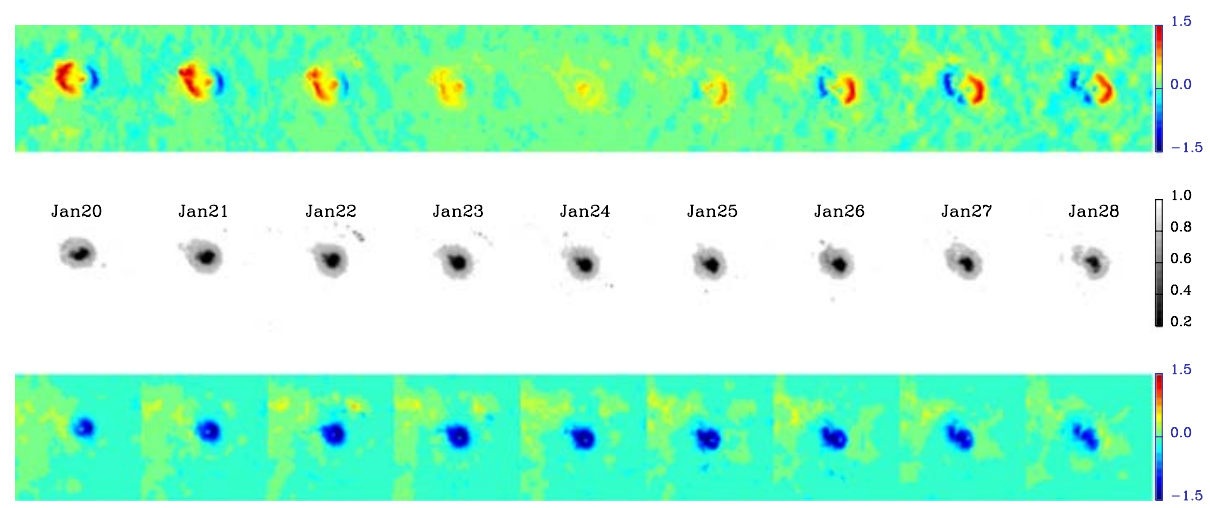

Figure 1 SOHO/MDI observations of the sunspot of Active Region 9787 during the period 20 - 28 January 2002. Shown are the daily averages of the line-of-sight Doppler velocity (top row), the intensity (middle row), and the line-of-sight magnetic field (bottom row). The color bars are in units of kilometers per second, relative intensity, and kilogauss, from top to bottom. 

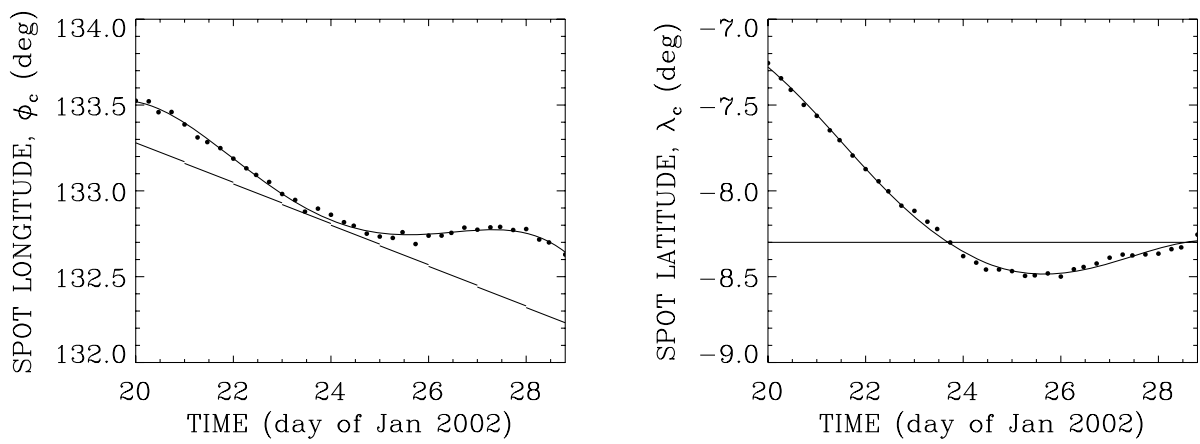

Figure 2 Coordinates of the center of the sunspot's umbra as a function of time. Left: Carrington longitude of the umbra (dots) and Carrington longitude at the center of the Postel projection (line segments). Right: Latitude of the umbra (dots) and latitude at the center of the Postel projection (horizontal line).

Figure 3 SOHO/MDI intensity image of AR 9787 averaged over nine days, after correcting for the proper motion of the sunspot. The intensity is measured relatively to the quiet-Sun value.

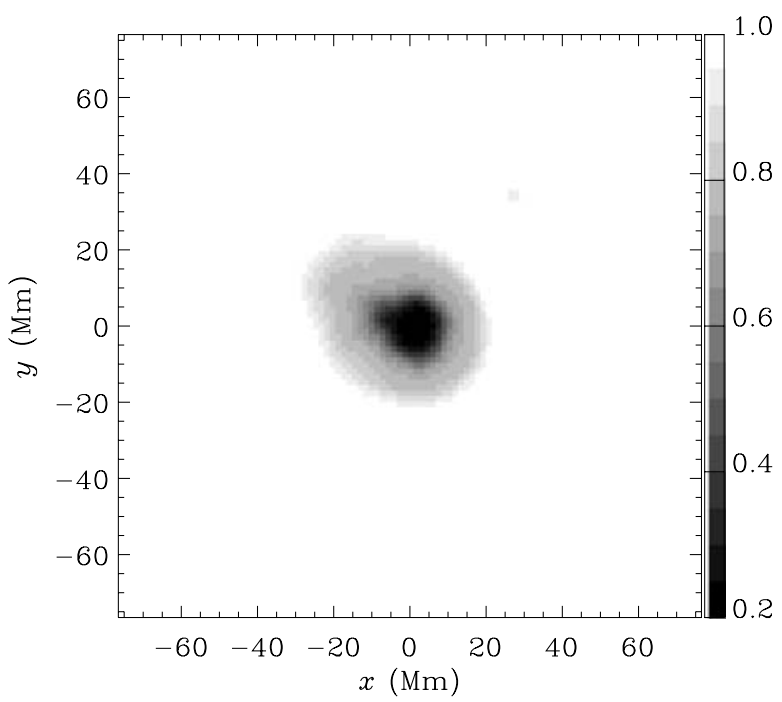

\section{Observed $f$-Mode Cross-Covariance Function}

We study sunspot AR 9787 using cross-covariances of the random wavefield, as is done in time-distance helioseismology (Duvall et al., 1993). The temporal cross-covariance between two points on the solar surface provides information about the Green's function between these two points. This interpretation has recently been shown to be correct in the case of homogeneously distributed random sources in an arbitrarily complex medium (Colin de Verdière, 2006; Gouédard et al., 2008).

In this paper, for the sake of simplicity, we wish to observe the propagation of $f$ modes through the sunspot. Thus we filter the Dopplergrams in 3D Fourier space to only keep the $f$ modes. Let us denote by $\phi(\mathbf{r}, t)$ the filtered Doppler velocity, where $\mathbf{r}$ is a position vector and $t$ is time. Rather than studying the cross-covariance of $\phi$ between two spatial points, we consider the cross-covariance between $\phi$ averaged over a great circle $\gamma$, denoted by $\bar{\phi}(\gamma, t)$, 
and $\phi$ measured at any other point $\mathbf{r}$ :

$$
C(\mathbf{r}, t)=\int_{0}^{T} \bar{\phi}\left(\gamma, t^{\prime}\right) \phi\left(\mathbf{r}, t+t^{\prime}\right) \mathrm{d} t^{\prime},
$$

where the effective integration time, $T$, is nine days, or 36 times six hours. Since averaging $\phi$ over $\gamma$ is equivalent to selecting only the horizontal wavevectors that are perpendicular to $\gamma$, this cross-covariance function gives us information about plane wave packets that propagate away from $\gamma$. In simple words, the cross-covariance at time lag $t$ tells us about the position of a wave packet, a time $t$ after it has left $\gamma$. In the rest of the paper we fix the distance between $\gamma$ and the center of the sunspot at $\Delta=40 \mathrm{Mm}$. Since $\Delta \gg \lambda$, where $\lambda \approx 5 \mathrm{Mm}$ is the dominant wavelength, the sunspot is in the far field of $\gamma$.

For any particular choice of orientation of $\gamma$, the computed cross-covariance is very noisy, thus explaining the need for some spatial averaging. Let us pick a reference great circle coincident with the meridian at a distance $40 \mathrm{Mm}$ eastward of the center of the sunspot. Because sunspot AR 9787 is almost rotationally invariant around its center, we can compute many equivalent cross-covariance functions corresponding to many different directions of the incoming wave packet, derotate these about the center of the sunspot so that they match the reference cross-covariance, and average them to reduce the noise. We have performed this averaging over all the directions of the incoming wavevectors, with a fine sampling of $1^{\circ}$. As seen in Figure 4, this enables us to reach a very good signal-to-noise level.

\section{Three-Dimensional MHD Simulation of Wave Propagation through a Sunspot}

\subsection{The Equations}

We use the ideal MHD equations, linearized about an arbitrary, inhomogeneous, magnetized atmosphere. We assume a local Cartesian geometry defined by horizontal coordinates $x$ and $y$, and the vertical coordinate $z$ increases upward. The level $z=0$ is assumed to correspond to the photosphere, as defined in model S (Christensen-Dalsgaard et al., 1996). Under the assumptions that gravitational acceleration $(g)$ is constant and that there is no background steady flow, the equation governing the wave-induced displacement vector $\xi$ is (e.g., Cameron, Gizon, and Daiffallah, 2007)

$$
\rho_{0} \partial_{t}^{2} \boldsymbol{\xi}=\mathbf{F}^{\prime}
$$

where

$$
\mathbf{F}^{\prime}=-\nabla P^{\prime}+\rho^{\prime} g \hat{\mathbf{z}}+\frac{1}{4 \pi}\left(\mathbf{J}^{\prime} \times \mathbf{B}_{0}+\mathbf{J}_{0} \times \mathbf{B}^{\prime}\right)
$$

is the linearized force acting on a fluid element (first order in $\boldsymbol{\xi}$ ), with the density, pressure, magnetic field, and electric current denoted by the symbols $\rho, P, \mathbf{B}$, and $\mathbf{J}$, respectively. In this paper the subscript 0 variables represent the steady inhomogeneous background atmosphere and the primed quantities represent the wave-induced perturbations. The system is closed by the following relations that define $\mathbf{F}^{\prime}$ in terms of $\boldsymbol{\xi}$ :

$$
\begin{aligned}
\rho^{\prime} & =-\nabla \cdot\left(\rho_{0} \xi\right), \\
P^{\prime} & =c_{0}^{2}\left(\rho^{\prime}+\boldsymbol{\xi} \cdot \nabla \rho_{0}\right)-\boldsymbol{\xi} \cdot \nabla P_{0}, \\
\mathbf{B}^{\prime} & =\nabla \times\left(\boldsymbol{\xi} \times \mathbf{B}_{0}\right), \\
\mathbf{J}^{\prime} & =\nabla \times \mathbf{B}^{\prime},
\end{aligned}
$$


Figure 4 Upper panel: The observed $f$-mode MDI cross-covariance function at zero time lag. The sunspot (black circle) is at the center of the Postel map, a distance $\Delta=40 \mathrm{Mm}$ from the meridian $\gamma$ (white line). Lower panel: The same cross-covariance at time lag $t=130$ minutes. To increase the signal-to-noise ratio, the cross-covariance was averaged over all wave directions. It is easy to see, by eye, that the waves are affected by their passage through the sunspot. The white rectangles show the size of the simulation box.
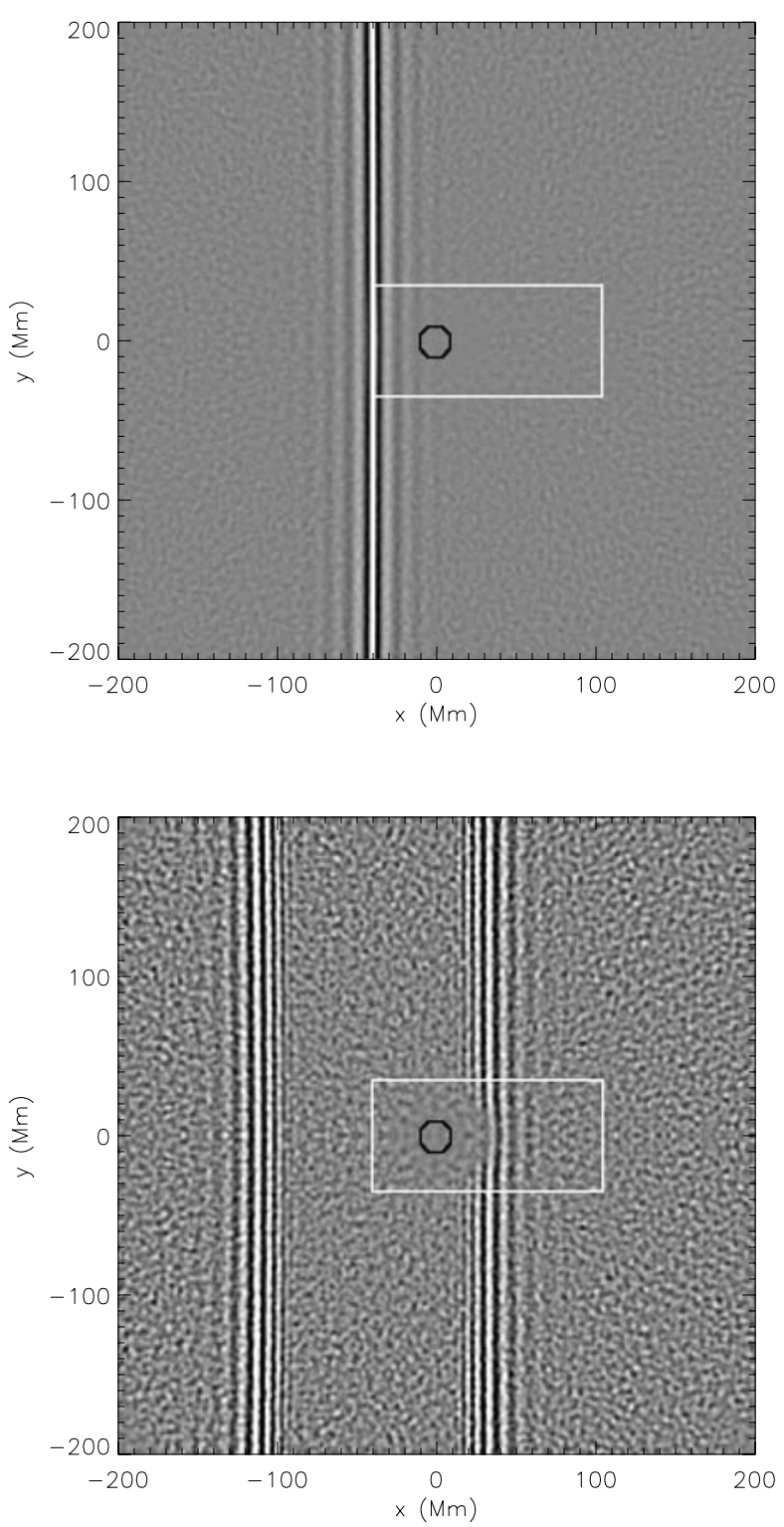

where $c_{0}$ is the background sound speed.

Although we have employed ideal MHD, the waves on the Sun are strongly attenuated, presumably as a result of scattering off the time-dependent granulation. Empirically, a solar mode with horizontal wavevector $\mathbf{k}$ decays as $\mathrm{e}^{-\gamma_{k} t}$, where $1 / \gamma_{k}$ is the e-folding lifetime at wavenumber $k=\|\mathbf{k}\|$. Introducing $\mathbf{v}^{\prime}$ as the wave velocity, for each horizontal Fourier mode we write

$$
\begin{aligned}
\rho_{0}\left(\partial_{t}+\gamma_{k}\right) \mathbf{v}^{\prime}(\mathbf{k}, z, t) & =\mathbf{F}^{\prime}(\mathbf{k}, z, t), \\
\left(\partial_{t}+\gamma_{k}\right) \xi(\mathbf{k}, z, t) & =\mathbf{v}^{\prime}(\mathbf{k}, z, t) .
\end{aligned}
$$


In these equations, any function $f(\mathbf{k}, z, t)$ refers to the spatial Fourier transform of $f(x, y, z, t)$. For $\gamma_{k}>0$ the system is damped. The eigenstates, pairs of $\left(\boldsymbol{\xi}, \mathbf{v}^{\prime}\right)$, are independent of $\gamma_{k}$, although the eigenvalues are naturally affected. This is the advantage of this approach. The attenuation that we have implemented acts only in the time domain and is suitable for our purpose, although in the Sun attenuation is more complicated. An alternate way of introducing this phenomenological time attenuation would have been to perform the ideal calculation and then impose the decay in the time domain post facto after the calculation has ended.

In this paper we focus on the $f$ modes (solar-surface gravity waves), for which the attenuation has been measured to be

$$
\gamma_{k}=\gamma_{*}\left(k / k_{*}\right)^{2.2},
$$

where $\gamma_{*} / \pi=100 \mu \mathrm{Hz}$ and $k_{*}=902 / R_{\odot}$ is a reference wavenumber (Duvall, Kosovichev, and Murawski, 1998; Gizon and Birch, 2002).

\subsection{The Code}

We use a modified version of the SLiM code, which is, apart from the modifications discussed in the following, described in Cameron, Gizon, and Daiffallah (2007). The code has been tested against analytic solutions; some of these tests are also described in detail in Cameron, Gizon, and Daiffallah (2007).

The present code includes two absorbing layers at the top and the bottom of the box. In the top layer above the temperature minimum we heavily damp the waves and systematically reduce the effect of the Lorentz force. This layer only affects waves that have escaped through the photosphere. Likewise the bottom layer damps the waves that propagate downward. The purpose of both layers is to minimize the effects of the boundaries, which would otherwise artificially reflect the waves.

An additional change has been to introduce the mode attenuation described in Section 4.1. Since we use a semispectral scheme the implementation was straightforward.

The scheme uses finite differences in the vertical direction, with 558 uniformly spaced grid points sampled at $\Delta z=25 \mathrm{~km}$. In the horizontal direction we use a Fourier decomposition with 200 modes in the $x$-direction and only 50 in the $y$-direction. The spatial sampling is $\Delta x=0.725 \mathrm{Mm}$ and $\Delta y=1.45 \mathrm{Mm}$. This seemingly low resolution is satisfactory because neither the initial wave packet nor the sunspot has any significant power at short wavelengths. The size of the simulation box is $145 \mathrm{Mm}$ long ( $x$-coordinate), $72.5 \mathrm{Mm}$ across ( $y$-coordinate), and $14 \mathrm{Mm}$ in depth (12.5 Mm below the photosphere). A typical run, such as the one from Section 5.3, takes approximately 14 days on a single-CPU core.

\subsection{Stabilizing the Quiet-Sun Atmosphere}

Our aim is to model the propagation of waves through the solar atmosphere in such a way as to allow a direct comparison with the observations. The most direct way of proceeding would be to use an existing solar-like atmosphere such as that of model S (ChristensenDalsgaard et al., 1996). This most direct approach is however unavailable when considering the full evolution of wave packets using numerical codes because both the solar and model $\mathrm{S}$ convection zones are convectively unstable.

Wave propagation through unstable atmospheres is difficult to study numerically because any numerical noise in the unstable modes grows exponentially and eventually dominates 
the numerical solution. The problem is then to stabilize the atmosphere while leaving it as solar-like as possible, at least in terms of the nature of the waves propagating through it.

The convective instability is easily understood by reference to Equation (5). We imagine a small vertical displacement of a blob of plasma and assume it is evolving slowly enough that it is in pressure balance with its surroundings. This implies $P^{\prime}=0$ and hence $c_{0}^{2} \rho^{\prime}=$ $\xi \cdot \nabla P_{0}-c_{0}^{2} \xi \cdot \nabla \rho_{0}$. For a vertically stratified atmosphere, this becomes $\rho^{\prime}=\xi_{z}\left(\partial_{z} P_{0}-\right.$ $\left.c_{0}^{2} \partial_{z} \rho_{0}\right) / c_{0}^{2}$. The atmosphere is convectively unstable if an upward displacement $\left(\xi_{z}>0\right)$ corresponds to a region of lowered density $\left(\rho^{\prime}<0\right)$; in such a case the fluid parcel is buoyant and accelerates upward. The atmosphere is convectively stable when $\xi_{z}$ and $\rho^{\prime}$ have the same sign, requiring $\left(\partial_{z} P_{0}-c_{0}^{2} \partial_{z} \rho_{0}\right) / c_{0}^{2}>0$, or equivalently

$$
\partial_{z} P_{0}>c_{0}^{2} \partial_{z} \rho_{0}
$$

We have the freedom to modify any combination of $P_{0}, c_{0}$, or $\rho_{0}$ to satisfy Equation (11). It is somewhat natural to regard these three quantities as being related through an equation of state or through the constraint that the atmosphere be hydrostatic; however, neither of these relationships is necessary in terms of the properties of the propagating waves. We choose $P_{0}$, $c_{0}$, and $\rho_{0}$ so that the wave speed is solar-like. Changing the sound speed would obviously have a major impact on the propagation of sound waves, and hence we have chosen to keep $c_{0}$ unchanged. Varying $\rho_{0}$ would have the effect of varying the distribution of the kinetic energy density of the different modes as a function of height, which would significantly change the sensitivity of wave packets to inhomogeneities; so we have also kept $\rho_{0}$ fixed. Thus we choose $P_{0}$ so that

$$
\partial_{z} P_{0}=\max \left\{0.9 c_{0}^{2} \partial_{z} \rho_{0}, \partial_{z} P_{\mathrm{u}}\right\},
$$

where $P_{\mathrm{u}}$ was the pressure distribution of the unstable atmosphere. Notice that $\partial_{z} P_{0}$ is negative so that the factor of 0.9 does indeed mean that Equation (11) is satisfied. The factor of 0.9 is possibly unnecessary, but it does little harm: Setting this factor to 1 should create stationary eigenmodes, and setting it to 0.9 introduces internal gravity modes, which propagate very slowly.

\subsection{An Example Oscillation Power Spectrum}

Since we have modified the atmosphere, we need to check whether it supports oscillations that are those of the Sun and model S in the absence of a sunspot. We chose initial conditions consisting of a "line source" of the form

$$
\xi_{z}=\mathrm{e}^{-x^{2} / 2 s^{2}} \mathrm{e}^{-\left(z-z_{0}\right)^{2} / 2 s^{2}} \quad \text { at } t=0,
$$

where $s=0.7 \mathrm{Mm}$ and $z_{0}=-250 \mathrm{~km}$ is an arbitrary height below the photosphere. All other wave perturbations, $\xi_{x}, \xi_{y}$, and $\mathbf{v}^{\prime}$, are zero at $t=0$. This disturbance encompasses many different modes allowing several ridges of the dispersion diagram to emerge. Figure 5 directly compares these ridges with the eigenfrequencies of model S (A.C. Birch, private communication). The $f$ modes and the acoustic modes $p_{1}, p_{2}$, and $p_{3}$ lie where they are expected for $k R_{\odot}>300$. It is also to be noted that there is a very low amplitude ridge (not visible on the plot) at frequencies below $1 \mathrm{mHz}$ that corresponds to internal gravity modes, which are not present in the Sun. They are an artifact of having made the system convectively stable as already explained, but they can be safely ignored. 
Figure 5 Power as a function of wavenumber and frequency for the example simulation described in Section 4.4 (no sunspot). The dashed lines correspond to the eigenfrequencies of the model $\mathrm{S}$ atmosphere.

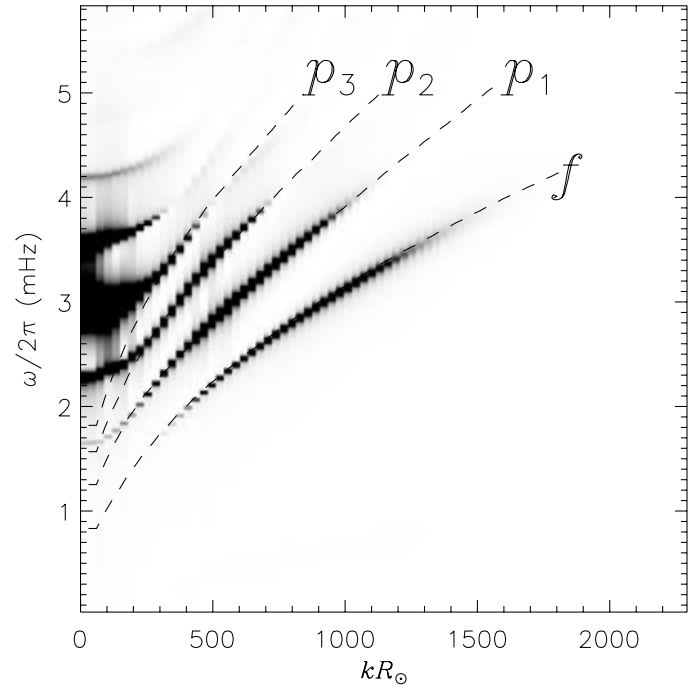

The simulations and model $\mathrm{S}$ eigenfrequencies differ strongly at wavenumbers less than $300 / R_{\odot}$. This difference occurs because our computational domain extends to only $12 \mathrm{Mm}$ below the solar surface. We have not tested a deeper box because the $f, p_{1}$, and $p_{2}$ modes look satisfactory in the range of wavelengths in which we are interested $\left(k>300 / R_{\odot}\right)$.

\subsection{A Parametric Sunspot Model}

In this section we will describe the sunspots that we have embedded in our atmosphere. We begin by noting that we embed the sunspot in the atmosphere before we stabilize it, to get the correct sound speed and density structures. We use $P_{\mathrm{u}}$ to denote the pressure of the atmosphere before it has been stabilized as previously described. The sunspot is modeled by an axisymmetric magnetic field $\mathbf{B}_{0}=\mathbf{B}_{0}(r, z)$, where $r$ is the horizontal radial distance from the sunspot axis. Within the part of the atmosphere described by model $\mathrm{S}$, the magnetic field is made hydrostatic in a standard way, by calculating the Lorentz force $\mathbf{L}=\left(\mathbf{J}_{0} \times \mathbf{B}_{0}\right) / 4 \pi$ and noting that the horizontal force balance then requires a horizontal pressure gradient: $\partial_{r} P_{\mathrm{u}}=\hat{\mathbf{r}} \cdot \mathbf{L}$. Since $P_{\mathrm{u}}$ is unaffected by the spot at large distances, we can integrate from infinity toward the center of the spot to find $P_{0}$. Having thus found $P_{u}$, we can find $\rho_{0}$ by the constraint of vertical force balance. In this case the gravitational force needs to balance both the vertical component of the Lorentz force as well as that of the pressure gradient. In principle given $\rho_{0}$ and $P_{0}$ the Saha-Boltzmann equations need to be solved to obtain the first adiabatic exponent $\left(\Gamma_{1}\right)$ and the sound speed $\left(c_{0}\right)$. However, for the purposes of this paper we have assumed that $\Gamma_{1}$ is a function of $z$ only and is unaffected by the sunspot. This assumption will be relaxed in future studies.

The procedure thus outlined can always be applied; however, in some cases the results will involve negative pressures or densities. Such solutions are of course unphysical and indicate that no hydrostatic solution exists for the given magnetic configuration and quietSun pressure stratification. The problem typically arises in the very upper layers of the box when the magnetic pressure is large. For example, a purely vertical flux tube with a magnetic pressure $P_{\mathrm{m}}$ cannot be in hydrostatic balance in an atmosphere with external pressure $P_{\text {ext }}<$ $P_{\mathrm{m}}$. In practice the Sun rapidly evolves to almost force-free field configurations above the solar surface. The role of the Lorentz force in structuring the atmosphere in the low- $\beta$ region 
is thus artificial as well as being responsible for the lack of equilibrium. It is also dynamically of minor importance to the waves since it is above both the acoustic cutoff and the layer where the acoustic and Alfvén wave speeds become equal. We have therefore adopted the approach (in the context of realistic photospheric magnetoconvection simulations of active regions; A. Nordlund, private communication) of scaling the Lorentz force in this region when constructing the background atmosphere. The scaling factor was chosen to be $1 /[1+$ $\left.B_{0}^{2} /\left(8 \pi P_{\mathrm{qs}}\right)\right]$, where $P_{\mathrm{qs}}$ is the quiet-Sun pressure in the absence of the spot. This scaling factor works, although a more conservative scaling will be tested in the future.

Above the model $\mathrm{S}$ atmosphere, the density and pressure fall rapidly and hydrostatic balance requires an extreme scaling of the Lorentz force. However, since we are not aiming to realistically model waves that reach these heights (we only want to damp them) this is acceptable. Instead of requiring force balance in this purely artificial region, we have chosen to put $P_{0}(x, y, z)=P_{\mathrm{qs}}(z) P_{0}\left(x, y, z_{0}\right) / P_{\mathrm{qs}}\left(z_{0}\right)$, where $z_{0}$ is the top of the model $\mathrm{S}$ atmosphere and $P_{\mathrm{qs}}(z)$ is the pressure stratification of the system in the absence of the spot (the quiet-Sun value). The density was treated similarly.

In this paper we follow Schlüter and Temesvary (1958), Deinzer (1965), and Schüssler and Rempel (2005) among others in concentrating on axisymmetric self-similar solutions. For this class of models, the vertical component of the magnetic field is assumed to satisfy

$$
B_{0 z}(r, z)=\mathcal{B}_{0} Q(r \sqrt{H(z)}), H(z)
$$

where the functions $Q$ and $H$ satisfy $Q(0)=H(0)=1$ but are otherwise arbitrary functions, and $\mathcal{B}_{0}$ is a scalar measure of the vertical-field strength at the surface. The usual practice (Solanki, 2003), which we adopt in this paper, is to assume $Q$ is a Gaussian,

$$
Q(r)=\mathrm{e}^{-(\ln 2) r^{2} / R_{0}^{2}},
$$

where $R_{0}$ is the half-width at half-maximum. We chose $R_{0}=10 \mathrm{Mm}$ to correspond to the observed value for sunspot AR 9787, since $R_{0}$ is the half-width at half-maximum of the model sunspot at the surface. The value of $R_{0}$ is fixed throughout this paper. For the function $H$ we chose the exponential function

$$
H(z)=\mathrm{e}^{z / \alpha},
$$

with $\alpha=6.25 \mathrm{Mm}$. This choice is rather arbitrary and will certainly be varied in the near future. In this paper we concentrate on varying $\mathcal{B}_{0}$, the peak magnetic field at the surface. Having thus prescribed the formula for $B_{0 z}(r, z)$, we determine $B_{0 r}(r, z)$ by the requirement that $\nabla \cdot \mathbf{B}_{0}=0$. Once we have such a hydrostatic solution, we adjust the pressure everywhere to make it convectively stable in the manner described earlier.

\section{Comparison between Simulations and Observations}

In this section we want to compare the observed $f$-mode cross-covariance from AR 9787 and the simulations. The computational domain is $-40<x<105 \mathrm{Mm},-36.25<y<$ $36.25 \mathrm{Mm}$, and $-12.5<z<1.5 \mathrm{Mm}$. The sunspot axis is $x=y=0$. The relationship of the computational box to the observations is shown by the white rectangle in Figure 4. 


\subsection{The Initial Conditions for the Simulation}

In this section we discuss the choice of the initial conditions for the simulation. The aim is to allow a direct comparison between the simulated wave field (Section 4) and the observed cross-covariance function (Section 3). Since the observed cross-covariance uses the $z$-component of the wave velocity as input data, we choose to use $v_{z}^{\prime}$ from the simulations for the comparison (which is also in accordance with a deeper interpretation of the cross-covariance; see Campillo and Paul, 2003). The vertical component of velocity of any $f$-mode wave packet propagating in the $+\hat{\mathbf{x}}$-direction in a horizontally homogeneous atmosphere is of the form

$$
v_{z}^{\prime}(x, y, z, t)=\operatorname{Re} \sum_{k} A_{k} \mathrm{e}^{k z} \mathrm{e}^{\mathrm{i} k\left(x-x_{0}\right)-\mathrm{i} \omega_{k} t-\gamma_{k} t},
$$

where $A_{k}$ are complex amplitudes, and $\exp (k z)$ and $\omega_{k}=\sqrt{g k}$ are the $f$-mode eigenfunction and eigenfrequency, respectively, at wavenumber $k$. We introduced the reference coordinate $x_{0}=-\Delta$, where $\Delta=40 \mathrm{Mm}$ is defined in the previous section as the distance between $\Gamma$ and the sunspot. Given the evolution (Equations (8) and (9)), this wave packet is uniquely determined by the initial conditions

$$
\begin{aligned}
\mathbf{v}^{\prime} & =\operatorname{Re} \sum_{k}(\mathrm{i} \hat{\mathbf{x}}+\hat{\mathbf{z}}) A_{k} \mathrm{e}^{k z+\mathrm{i} k\left(x-x_{0}\right)}, \\
\boldsymbol{\xi} & =\operatorname{Re} \sum_{k}(-\hat{\mathbf{x}}+\mathrm{i} \hat{\mathbf{z}}) \omega_{k}^{-1} A_{k} \mathrm{e}^{k z+\mathrm{i} k\left(x-x_{0}\right)} .
\end{aligned}
$$

Thus our problem is reduced to fixing the amplitudes $A_{k}$. In this study, the amplitudes $A_{k}$ are real (all waves are in phase at $x=x_{0}$ and $t=0$ ) and are shown in Figure 6. This choice was simply the result of requiring that the simulated $v_{z}^{\prime}(x, y, z=0, t)$ and the cross-covariance look approximately the same, in the far field and in the absence of the sunspot. As will be shown in the next section, this spectrum of wavenumbers is sufficiently accurate for the present study. A more systematic analysis is planned. One important difficulty that arises is the existence of background solar noise, which is not easy to model and has been ignored in Equation (17).

Figure 6 The initial distribution of $f$-mode amplitudes used in the simulations, $A_{k}$, as a function of $k R \odot$.

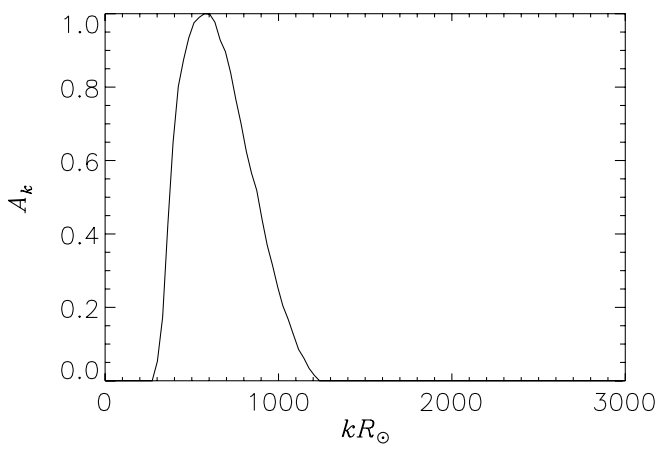




\subsection{Magnetoacoustic Waves}

First we consider a sunspot model with $\mathcal{B}_{0}=3 \mathrm{kG}$. The simulation enables us to understand what happens below the solar surface. The theory of the interaction of solar waves with sunspots had been developed by using ray theory and two-dimensional numerical simulations (e.g., Cally, 2007; Bogdan et al., 1996). We find what appears to be very similar physics in our three-dimensional simulations: The strong "absorption" of the $f$ modes is consistent with partial conversion of the incoming waves into slow magnetoacoustic waves, which propagate along the field lines. This can be seen in Figure 7. The downward propagating slow magnetoacoustic modes experience a decrease in their speed as they propagate downward and are therefore shifted to increasingly short wavelengths. Mode conversion is a very robust feature of this type of simulation. We note that the slow magnetoacoustic waves are much easier to see in the $x$-component of wave velocity than in $v_{z}^{\prime}$, which is why Figure 7 shows the former.

Since we are strongly damping short wavelengths, these magnetoacoustic modes rapidly decay. Any upward propagating wave encounters the damping buffer situated above the photosphere and is also damped. In principle this decay is unphysical in both instances; however, because neither the downward nor upward propagating waves return to the surface this is not undesirable.

\subsection{Waveforms}

In this section we compare the simulated $v_{z}^{\prime}$ at $z=0$ with the observed cross-covariance. We emphasize that at the moment this comparison is not expected to be appropriate in the immediate vicinity of the sunspot.

Figure 8 shows such a comparison between simulations and observations for times $t=$ 40, 100, and 130 minutes. The peak field strength used in this simulation was $\mathcal{B}_{0}=3 \mathrm{kG}$ and the radius was $R_{0}=10 \mathrm{Mm}$. The comparison appears to be very good at time $t=$ 130 minutes, at which time the wave packet has completely traversed the sunspot. At this time, all aspects of the waveform - amplitudes (including "absorption"), phases, and spatial spectrum - would appear to have been approximately reproduced by the simulation. The match appears to be poorer for $t=40$ minutes; this will be discussed in the following.

To be more precise, however, the match between the simulations and observations must be better quantified. To reduce the observational noise, we have averaged the crosscovariance function in the $y$-direction over two bands. Both bands, shown in Figure 9, are 14.5 Mm wide in the $y$-direction. The bands are labeled $\mathrm{A}$ and $\mathrm{B}$ and centered around $y=0$ and $y= \pm 29 \mathrm{Mm}$. Band A is centered on the spot, and band B acts as a reference. The simulations are averaged in the same manner for comparison with the observations.

In Figure 10 we have plotted both the simulation ( $v_{z}^{\prime}$, thick red lines) and the observed cross-covariance ( $C$, thin blue lines). In the top panels we compare the wave propagation at the edge of the computational box (i.e., in band B). This part of the wave is little affected by the sunspot and the match between the simulation and observation is quite good since the initial amplitude spectrum $A_{k}$ was chosen accordingly. In the lower panels of Figure 10 we see the results for the waves passing through the spot (i.e., band A). First we notice that the wave amplitude is remarkably well reproduced in the simulations, meaning that the observed wave "absorption" is consistent with mode conversion. For $t=130$ minutes, after the waves have crossed the sunspot, there is no appreciable phase shift between the simulation and the observations. The match, however, is somewhat worse at $t=40$ minutes and there is an obvious phase shift between the two waveforms with the observations lagging behind the 

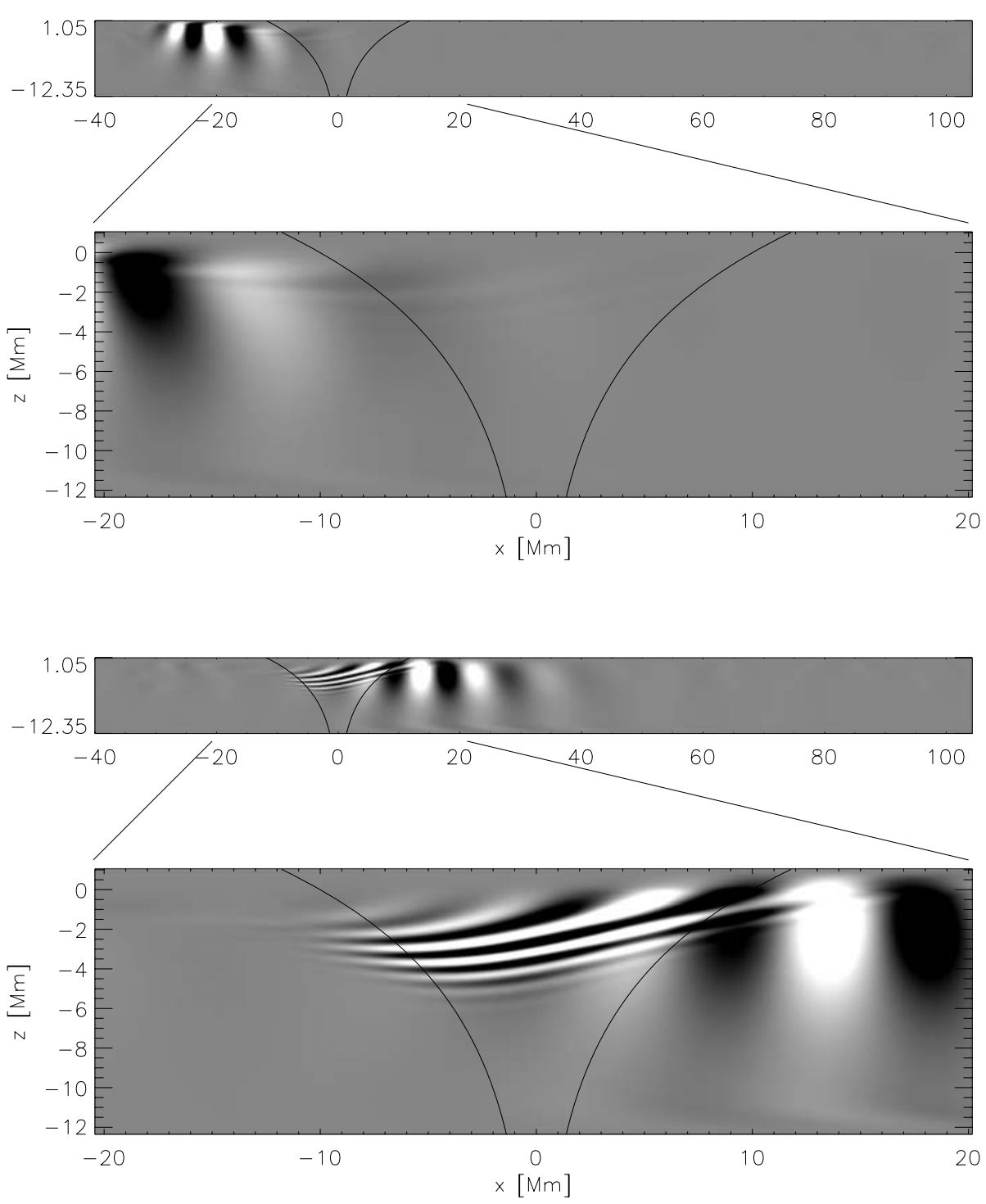

Figure 7 Plot showing $\rho_{0} v_{x}^{\prime}$ in the $x-z$ plane, through the sunspot axis. The upper frames are for time $t=34$ minutes (before the $f$-mode wavepacket crosses the sunspot) and the lower frames are for $t=84$ minutes (afterward). The black curves with equation $B_{z}(r, z)=B_{z}(r=0, z) / 2$ give an estimate of the "width" of the sunspot. The conversion of the incoming $f$ modes into slow magnetoacoustic modes is evident in the lower frames.

simulations. Given the value of the phase shift, we suspect that it is due to the effect of the moat flow. The moat flow is a horizontal outflow from the sunspot, which we have measured by tracking the small moving magnetic features. The observed moat velocity (averaged over nine days) has a peak value of $230 \mathrm{~m} \mathrm{~s}^{-1}$ at a distance of $25 \mathrm{Mm}$ from the center of the sunspot and vanishes at a distance of $45 \mathrm{Mm}$. The solar waves moving through the flow are first slowed down (against the flow) and later sped up again (with the flow). We have not 

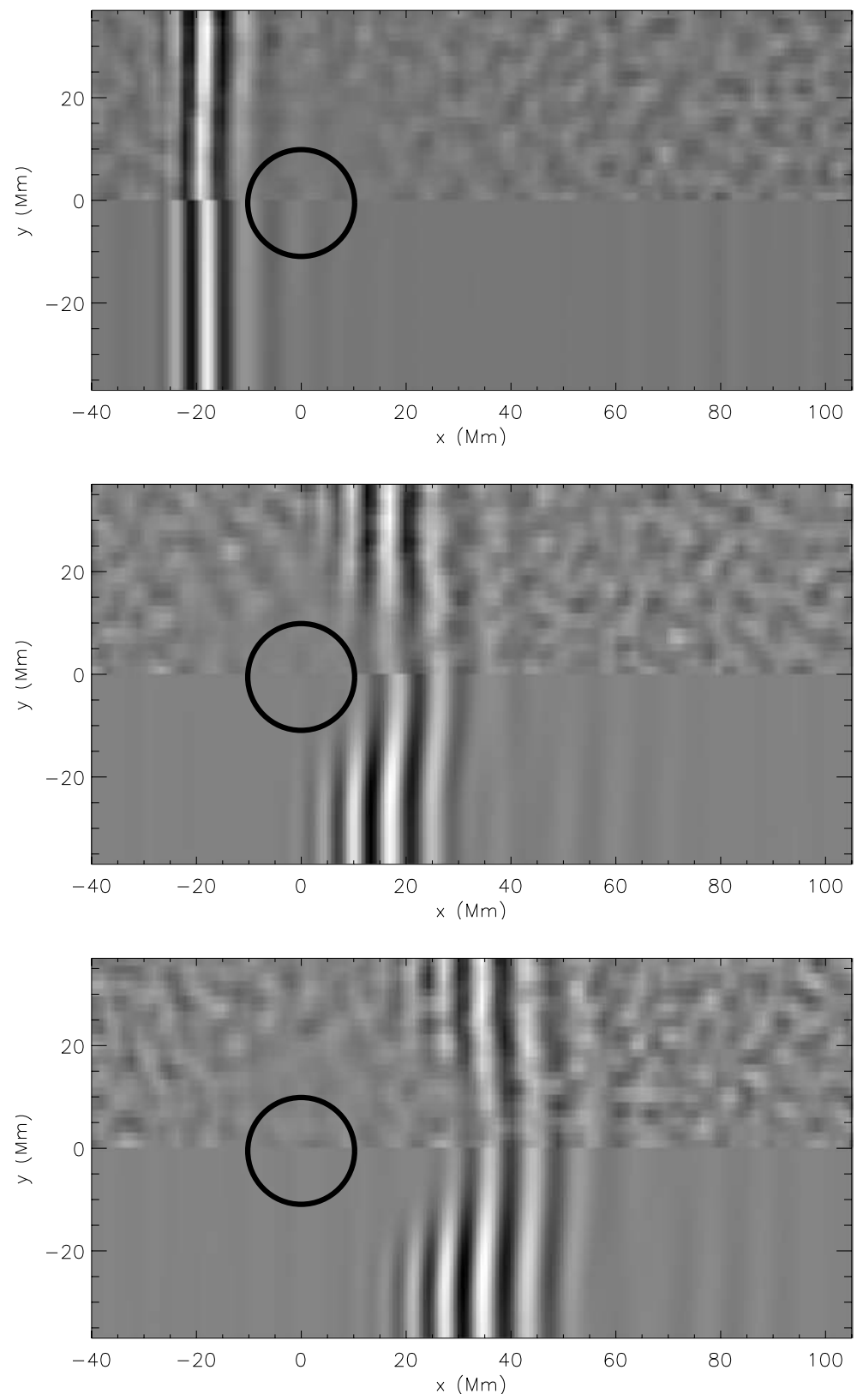

Figure 8 Comparison of the simulated vertical velocity and the observed cross-covariance. In each panel the upper frame shows the observed cross-covariance and the bottom panel the simulated wavepacket. The panels correspond to $t=40$ minutes, $t=100$ minutes, and $t=130$ minutes, from top to bottom. The black circles of radius $R_{0}=10 \mathrm{Mm}$ indicate the location of the sunspot.

modeled this effect yet. It is reassuring, however, to see that the Doppler shift caused by the moat appears to have disappeared at $t=130$ minutes. 


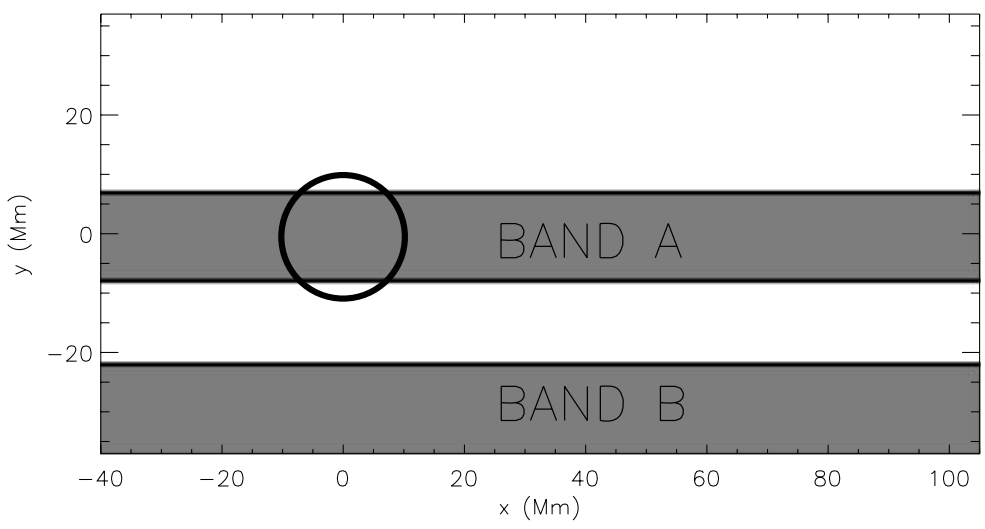

Figure 9 Sketch of the bands over which the data have been averaged in the $y$-direction in later plots. Band A is centered on the spot, and band B acts as a reference.

Reiterating, we find that, by using numerical simulations, it appears that seismic signatures can be followed through their passage across the spot.

\section{Constraining B}

The solutions shown thus far have been for a model sunspot with a peak vertical-field strength $\mathcal{B}_{0}=3 \mathrm{kG}$ at the surface $z=0$. The match is good, which raises the question of whether other field strengths would match as well. The answer to this question can be seen in Figure 11, where we show the comparison between the simulations and observations for different field strengths, $\mathcal{B}_{0}=2 \mathrm{kG}$ and $\mathcal{B}_{0}=2.5 \mathrm{kG}$. The comparison is made some time after the wave packet has passed through the sunspot, so the issue of the moat flow does not arise. At this stage we restrict ourselves to commenting that qualitatively the match is best, in phase and amplitude, for $\mathcal{B}_{0}=3 \mathrm{kG}$; for the other values of $\mathcal{B}_{0}$ there is an apparent phase mismatch along $y=0$ and the amplitude of that part of the wave passing through the spot is not sufficiently damped. The quantification of the "goodness of fit" between the simulation and observations, which will allow a more accurate determination of the field strength, will be the subject of a future study.

\section{Discussion}

We have performed three-dimensional MHD simulations of waves propagating through sunspot models. The computations are set up in such a way as to allow comparing observed cross-covariances (except in the immediate vicinity of the sunspot). The parameters of the sunspot model can be chosen in such a way that its helioseismic signature is in good agreement with helioseismic observations of sunspot AR 9787.

A qualitative study using $f$ modes has enabled us to place a constraint, $\mathcal{B}_{0} \geq 3 \mathrm{kG}$, on the sunspot's near-surface field strength. The remaining differences reflect real differences between the model and the observed sunspot, such as the moat flow.

The model atmosphere that we have constructed also supports $p$ modes with a dispersion relation that is very close to that of the Sun. Obviously, the $p$ modes in combination with the 

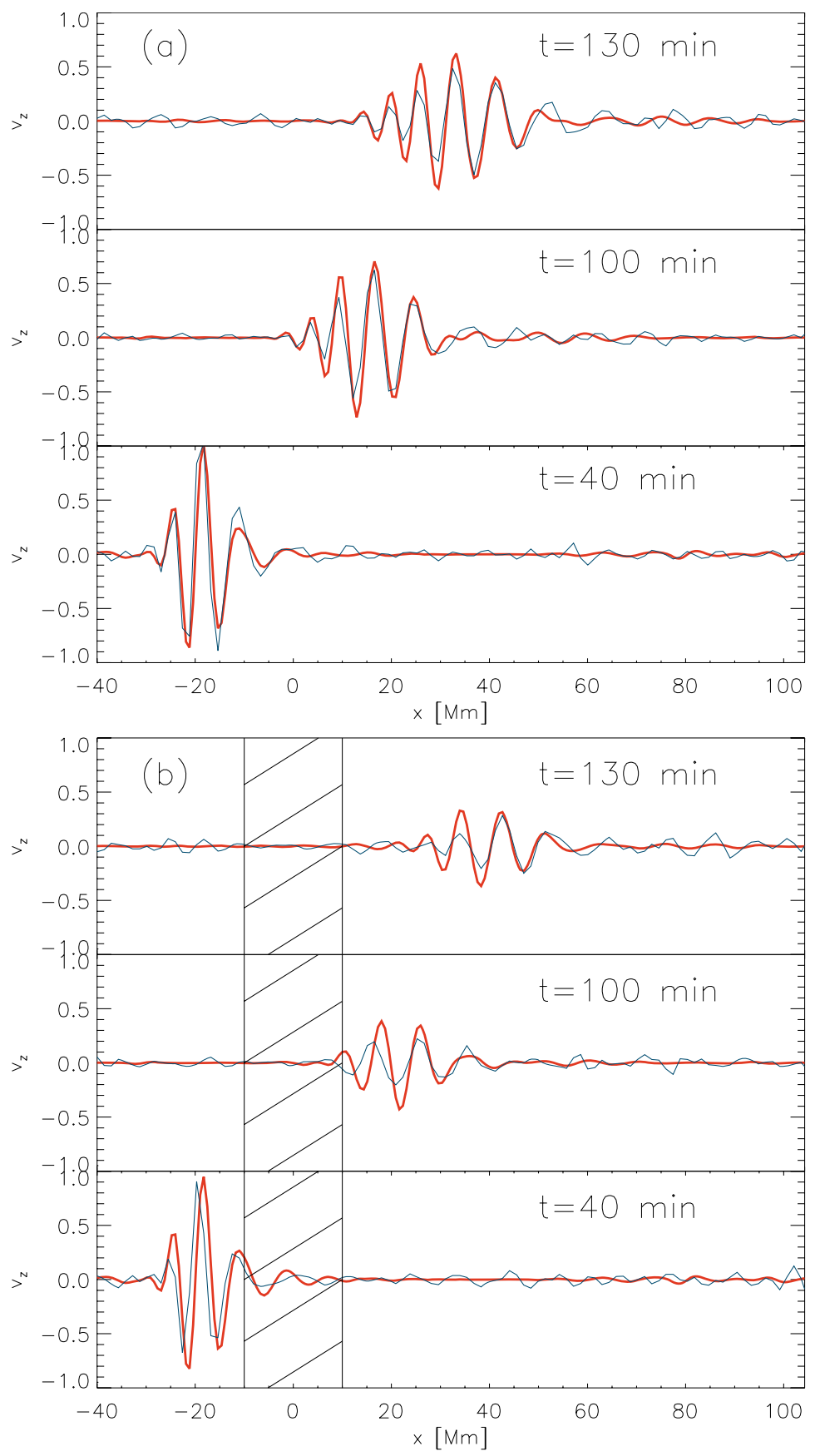

Figure 10 Top panels: The simulation ( $v_{z}^{\prime}$, thick red lines) and the observed cross-covariance $(C$, thin blue lines) averaged in the $y$-direction across band B (reference), at three different times $(t)$. Bottom panels: The simulation $\left(v_{z}^{\prime}, \mathcal{B}_{0}=3 \mathrm{kG}\right.$, thick red lines) and observed cross-covariance $(C$, thin blue lines) averaged in the $y$-direction across band $\mathrm{A}$, at three different times $(t)$. The effect of the sunspot is very easily seen, by comparing with band $\mathrm{B}$. The stripes indicate the location of the sunspot umbra. 

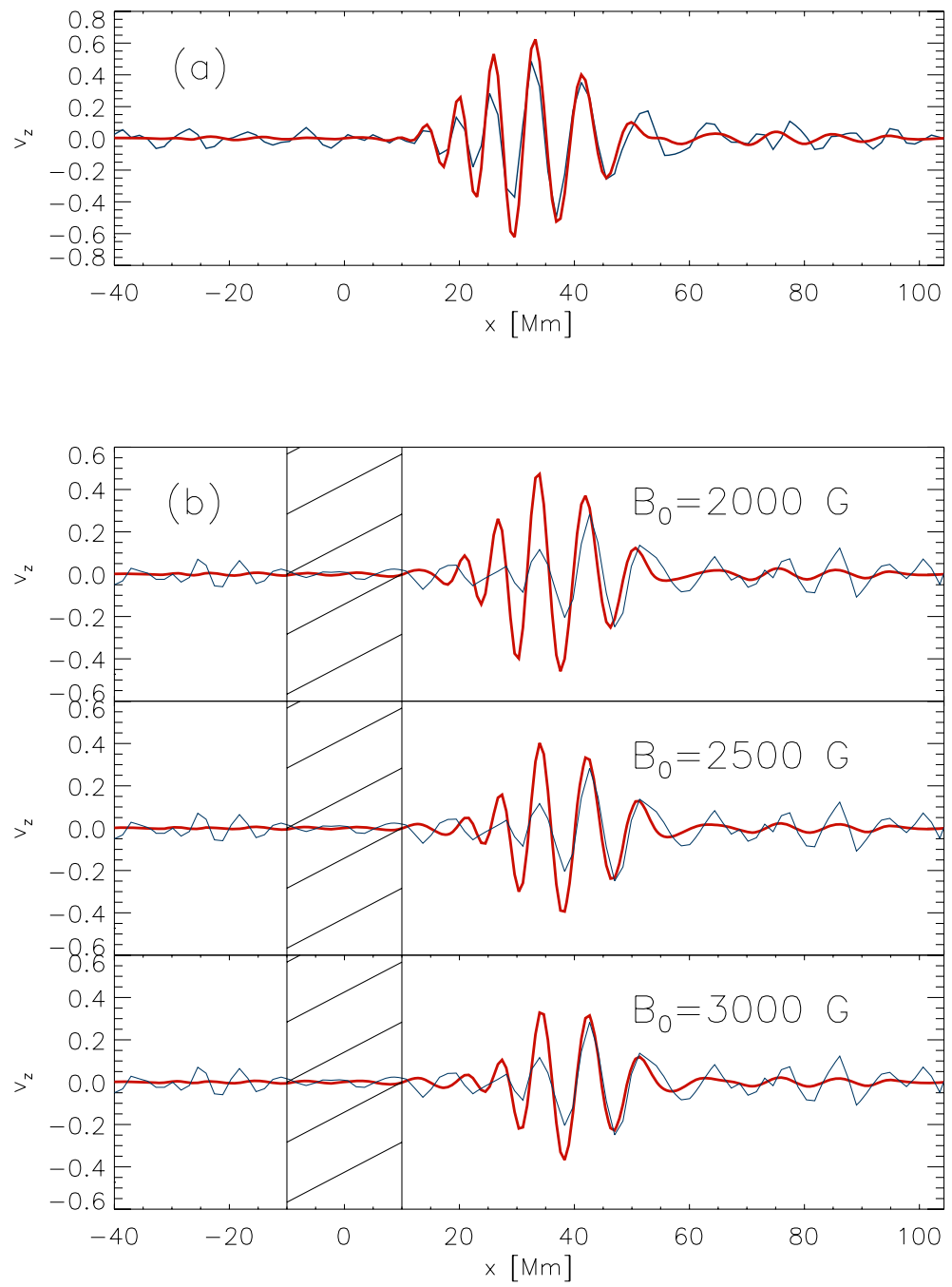

Figure 11 Simulated vertical velocity (thick red lines) and observed cross-covariance (thin blue lines) at $t=130$ minutes (a) at the edge of the domain (large $y)$ and (b) passing through the sunspot $(y=0)$. The three panels correspond to simulations using $\mathcal{B}_{0}=2,2.5$, and $3 \mathrm{kG}$. It is seen that the simulation with $\mathcal{B}_{0}=3 \mathrm{kG}$ provides the best match in terms of both amplitude and phase. The stripes indicate the location of the sunspot umbra.

$f$ modes should enable us to place more substantial constraints on the subsurface structure of the sunspot. In particular, it should be possible to simultaneously constrain the magnetic field strength $\mathcal{B}_{0}$, the sunspot radius $R_{0}$, and the magnetic field inclination (controlled by the parameter $\alpha$ ). Of course, the parametric sunspot model that we have considered in this paper is just one particular model, we plan to consider other types of sunspot models in the future.

In summary, we believe that we have shown that the full-waveform modeling of sunspots is feasible. 
Acknowledgements SOHO is a project of international collaboration between ESA and NASA. We are grateful to Manfred Schüssler for insightful discussions. This work was supported in part by the European Helio- and Asteroseismology Network (HELAS) funded by the European Union.

Open Access This article is distributed under the terms of the Creative Commons Attribution Noncommercial License which permits any noncommercial use, distribution, and reproduction in any medium, provided the original author(s) and source are credited.

\section{References}

Birch, A., Kosovichev, A.G., Price, G.H., Schlottmann, R.B.: 2001, Astrophys. J. 561, L229.

Bogdan, T.J., Cally, P.S.: 1997, Proc. Roy. Soc. London, Ser. A 453, 919.

Bogdan, T.J., Hindman, B.W., Cally, P.S., Charbonneau, P.: 1996, Astrophys. J. 465, 406.

Braun, D.C.: 1995, Astrophys. J. 451, 859.

Braun, D.C., Duvall, T.L. Jr., LaBonte, B.J.: 1987, Astrophys. J. 319, L27.

Cally, P.: 2000, Solar Phys. 192, 395.

Cally, P.: 2007, Astron. Nachr. 328, 286.

Cally, P., Bogdan, T.: 1993, Astrophys. J. 402, 721.

Cally, P., Bogdan, T.: 1997, Astrophys. J. 486, L67.

Cally, P., Goossens, M.: 2008, Solar Phys. DOI: 10.1007/s11207-007-9086-3.

Cally, P., Bogdan, T., Zweibel, E.G.: 1994, Astrophys. J. 437, 505.

Cally, P., Crouch, A., Braun, D.C.: 2003, Mon. Not. Roy. Astron. Soc. 346, 381.

Cameron, R., Gizon, L., Daiffallah, K.: 2007, Astron. Nachr. 328, 313.

Campillo, M., Paul, A.: 2003, Science 299, 547.

Colin de Verdière, Y.: 2006, http://fr.arxiv.org/abs/math-ph/0610043/.

Christensen-Dalsgaard, J., Däppen, W., Ajukov, S.V., Anderson, E.R., Antia, H.M., Basu, S., Baturin, V.A., Berthomieu, G., Chaboyer, B., Chitre, S.M., Cox, A.N., Demarque, P., Donatowicz, J., Dziembowski, W.A., Gabriel, M., Gough, D.O., Guenther, D.B., Guzik, J.A., Harvey, J.W., Hill, F., Houdek, G., Iglesias, C.A., Kosovichev, A.G., Leibacher, J.W., Morel, P., Proffitt, C.R., Provost, J., Reiter, J., Rhodes, E.J. Jr., Rogers, F.J., Roxburgh, I.W., Thompson, M.J., Ulrich, R.K.: 1996, Science 272, 1286.

Couvidat, S., Birch, A.C., Kosovichev, A.G.: 2006, Astrophys. J. 640, 516.

Crouch, A.D., Cally, P.S., Charbonneau, P., Braun, D.C., Desjardins, M.: 2005, Mon. Not. Roy. Astron. Soc. 363, 1188.

Deinzer, W.: 1965, Astrophys. J. 141, 548.

Duvall, T.L. Jr., Kosovichev, A.G., Murawski, K.: 1998, Astrophys. J. 505, L55.

Duvall, T.L. Jr., Jefferies, S.M., Harvey, J.W., Pomerantz, M.A.: 1993, Nature 362, 430.

Gizon, L., Birch, A.C.: 2002, Astrophys. J. 571, 966.

Gouédard, P., Stehly, L., Brenguier, F., Campillo, M., Colin de Verdière, Y., Larose, E., Margerin, L., Roux, Ph., Sánchez-Sesma, F.J., Shapiro, N., Weaver, R.: 2008, Geophys. Prospect. accepted.

Hanasoge, S.M., Duvall, T.L. Jr.: 2007, Astron. Nachr. 328, 319.

Hanasoge, S.M., Duvall, T.L. Jr., Couvidat, S.: 2007, Astrophys. J. 664, 1243.

Hanasoge, S.M., Larsen, R.M., Duvall, T.L. Jr., DeRosa, M.L., Hurlburt, N.E., Schou, J., Roth, M., Christensen-Dalsgaard, J., Lele, S.K.: 2006, Astrophys. J. 648, 1268.

Hindman, B.W., Zweibel, E.G., Cally, P.: 1996, Astrophys. J. 459, 760.

Khomenko, E., Collados, M.: 2006, Astrophys. J. 653, 739.

Kosovichev, A.G., Duvall, T.L. Jr., Scherrer, P.H.: 2000, Solar Phys. 192, 159.

Lindsey, C., Braun, D.C.: 1997, Astrophys. J. 485, 895.

Parker, E.: 1979, Astrophys. J. 230, 905.

Rosenthal, C.S., Julien, K.A.: 2000, Astrophys. J. 532, 1230.

Schüssler, M., Rempel, M.: 2005, Astron. Astrophys. 441, 337.

Schlüter, A., Temesvary, S.: 1958. In: Lehnert, B. (ed.) Electromagnetic Phenomena in Cosmical Physics, IAU Symp. 6, Cambridge University Press, Cambridge, 263.

Schunker, H., Cally, P.S.: 2006, Mon. Not. Roy. Astron. Soc. 372, 551.

Solanki, S.: 2003, Astron. Astrophys. Rev. 11, 153.

Spruit, H.C.: 1991. In: Toomre, J., Gough, D.O. (eds.) Challenges to Theories of the Structure of Moderate Mass Stars, Lecture Notes in Physics 388, Springer, Berlin, 121.

Spruit, H.C., Bogdan, T.: 1992, Astrophys. J. 391, L109.

Zhao, J., Kosovichev, A.G., Duvall, T.L. Jr.: 2001, Astrophys. J. 557, 384.

Zhao, J., Georgobiani, D., Kosovichev, A.G., Benson, D., Stein, R.F., Nordlund, Å.: 2007, Astrophys. J. 659, 848 